\title{
Lenalidomide plus R-CHOP21 in newly diagnosed diffuse large B-cell lymphoma (DLBCL): long-term follow-up results from a combined analysis from two phase 2 trials
}

\author{
A. Castellino ${ }^{1,2}$, A. Chiappella ${ }^{1}$, B. R. LaPlant ${ }^{2}$, L. D. Pederson $0^{2}$, G. Gaidano ${ }^{3}$, W. R. Macon², G. Inghirami ${ }^{4}$, C. B. Reeder ${ }^{5}$,

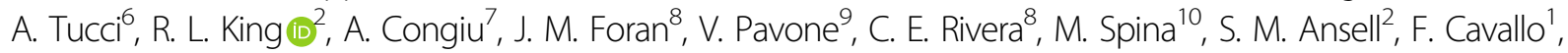 \\ A. L. Molinari ${ }^{11}$, Giovannino Ciccone ${ }^{12}$, T. M. Habermann², T. E. Witzig ${ }^{2}$, U. Vitolo ${ }^{1}$ and G. S. Nowakowski
}

\begin{abstract}
Lenalidomide-RCHOP (R2-CHOP21) has been shown to be safe and effective in patients with untreated diffuse large Bcell lymphoma (DLBCL). The aim of this analysis is to report long-term outcome and toxicities in newly diagnosed DLBCL patients who received R2-CHOP21 in two independent phase 2 trials, conducted by Mayo Clinic (MC) and Fondazione Italiana Linfomi (FIL). All patients received R-CHOP21 plus lenalidomide. Long-term progression-free survival (PFS), time to progression (TTP), overall survival (OS) and late toxicities and second tumors were analyzed. Hundred and twelve patients (63 MC, 49 FIL) were included. Median age was 69 years, 88\% were stage III-IV. At a median follow-up of 5.1 years, 5y-PFS was 63.5\%, 5y-TTP 70.1\% and 5y-OS 75.4\%; according to cell of origin (COO): 5 PFS $52.8 \%$ vs $64.5 \%, 5 y-T$ TP $61.6 \%$ vs $69.6 \%$ and $5 y-O S 68.6 \%$ vs $74.1 \%$ in germinal center (GCB) vs non-GCB respectively. Four patients experienced grade $4-5$ late toxicities. Grade $\leq 3$ toxicities were infections $(N=4)$, thrombosis $(N=1)$ and neuropathy $(N=3)$. Seven seconds tumors were observed. Long-term follow-up demonstrates that R2-CHOP21 efficacy was maintained with high rates of PFS, TTP, and OS. Lenalidomide appears to mitigate the negative prognosis of non-GCB phenotype. Incidence of therapy-related secondary malignancies and late toxicities were low.
\end{abstract}

\section{Introduction}

The addition of the anti-CD20 monoclonal antibody rituximab, to a chemotherapy regimen with cyclophosphamide, doxorubicin, vincristine, and prednisone ( $\mathrm{R}$ CHOP) dramatically improved the outcome of patients affected by diffuse large . B-cell lymphoma (DLBCL) and became the standard of treatment ${ }^{1-4}$. However, about $40 \%$ of patients relapse or do not respond to initial

\footnotetext{
Correspondence: A. Castellino (acastellino@cittadellasalute.to.it)

(Castellino.Alessia@mayo.edu)

${ }^{1}$ Azienda Ospedaliero Universitaria Città della Salute e della Scienza di Torino, Torino, Italy

${ }^{2}$ Mayo Clinic, Rochester, MN, USA

Full list of author information is available at the end of the article.

These authors contributed equally: Alessia Castellino, Annalisa Chiappella
}

chemoimmunotherapy, and patients with relapsed DLBCL have a poor prognosis ${ }^{5,6}$

Attempts to improve the efficacy of front-line therapy have included dose-dense and dose-intensified regimens followed by autologous stem cell transplantation $(\mathrm{ASCT})^{7-10}$, the use of different induction schedules ${ }^{11}$, early intensification of rituximab administration ${ }^{12}$, or the substitution of obinutuzumab for rituximab ${ }^{13}$. However they did not improve the outcome of DLBCL patients ${ }^{14}$.

The biological complexity of DLBCL suggests that a tailored therapeutic approach based on the biological and molecular signature might be a promising strategy. Geneexpression profiling (GEP) identified two major DLBCL subtypes: the germinal center B-cell like (GCB) and the 
activated B-cell like (ABC) also refered to as $\mathrm{GCB}$ and non-GCB in immunohistochemistry (IHC)-based subtyping ${ }^{15,16}$. DLBCLs with ABC (non-GCB) phenotype are associated with an unfavorable outcome when treated with standard R-CHOP ${ }^{17}$.

Lenalidomide is an immunomodulatory drug (ImiD) that demonstrates significant activity in relapsed/refractory lymphomas in monotherapy and in combination with rituximab $^{18,19}$. In xenograft models of DLBCL, lenalidomide demonstrated major clinical activity on $\mathrm{ABC}$-subtype, in which there was downregulation of B-cell receptor-dependent NF-kB through an inhibition of the transcription factor interferon regulatory factor 4 (IRF4) and cerebron ${ }^{20}$. Lenalidomide was subsequently investigated as front-line treatment of DLBCL in combination with standard R-CHOP chemoimmunotherapy administered every 21 days (R2-CHOP21). Two phase I/II studies, conducted by Mayo Clinic (MC078E trial) ${ }^{21,22}$ and by Fondazione Italiana Linfomi (FIL, REAL07 trial) ${ }^{23,24}$ reported that the combination regimen $\mathrm{R} 2-\mathrm{CHOP} 21$ is feasible and effective, with an overall response rate (ORR) greater than $90 \%$. These early results have led to two currently ongoing randomized trials ${ }^{25,26}$. However, longterm efficacy and safety of this regimen are not well defined. Here, we report the results of a combined analysis on a long-term follow-up of the efficacy and safety in newly diagnosed DLBCL patients who received R2CHOP21 in these two independent single-arm phase 2 studies.

\section{Methods}

\section{Study design and participants}

This analysis included all patients with newly diagnosed histologically-confirmed CD20+ DLBCL that were enrolled in two R2-CHOP21 phase 2 trials; one conducted by Mayo Clinic $\left(\mathrm{MC} 078 \mathrm{E}\right.$ trial $^{22}$ ) between September 2008 and August 2013, and one conducted by Fondazione Italiana Linfomi (REAL07 trial ${ }^{24}$ ) between April 2010 and June 2011.

MC078E was an investigator-initiated, open-label, single-arm phase I/II study, while REAL07 was an openlabel, multicentre phase I/II trial that was conducted in 13 centers in Italy and one center in Germany.

Patients in phase II and those from phase I that were administered the maximum tolerated dose of lenalidomide were included in this present long-term analysis.

The main differences between the two populations were that the MC078E trial included all patients older than 18 years without an upper age limit and all International Prognostic Index (IPI) risk scores, while the REAL07 trial focused on patients aged between 60 and 80 years old and included only patients with IPI score $\geq 2$, excluding low risk IPI score cases. Other inclusion criteria for both studies were similar and included the following: Ann
Arbor Stage II-IV; measurable disease with at least one lesion $\geq 1.5 \mathrm{~cm}$ in a single diameter by CT; Eastern Cooperative Oncology Group (ECOG) PS 0-2; estimated cardiac ejection fraction $\geq 45 \%$ (MUGA scan or echocardiogram); and preserved organ functions ${ }^{22,24}$. Main exclusion criteria included the following: presence of central nervous system (CNS) involvement at diagnosis, $\mathrm{HIV}, \mathrm{HCV}$, and $\mathrm{HBV}$ infection, history of life-threatening or recurrent thrombosis and/or embolism, unless they were receiving anticoagulant therapy during the protocol treatment; history of any neoplasia in the previous 3 years.

\section{Procedures}

All patients included in the analysis received standard R-CHOP21 treatment in association with lenalidomide administered in different schemes in the MC078E and REAL07 trials.

Both studies utilized standard R-CHOP21 therapy (rituximab $375 \mathrm{mg} / \mathrm{m}^{2}$, cyclophosphamide $750 \mathrm{mg} / \mathrm{m}^{2}$, doxorubicin $50 \mathrm{mg} / \mathrm{m}^{2}$, vincristine $1.4 \mathrm{mg} / \mathrm{m}^{2}$ (capped at $2 \mathrm{mg}$ ), all on day 1 , prednisone $100 \mathrm{mg} / \mathrm{m}^{2}$ (MC078E) or $40 \mathrm{mg} / \mathrm{m}^{2}$ (FIL-REAL07) per day on days 1 through 5 , given every 21 days).

In the MC078E trial, lenalidomide was administered at a dose of $25 \mathrm{mg} /$ day for 10 days/cycle (MTD determined in the phase I trial ${ }^{21}$ ), whereas in the REAL07 study, lenalidomide dose was $15 \mathrm{mg} /$ day for 14 days/cycle (MTD determined by phase 1 stydy ${ }^{23}$ ). Accordingly, the total cumulative doses of lenalidomide in both studies were similar: $250 \mathrm{mg} /$ cycle and $210 \mathrm{mg} /$ cycle in Mayo Clinic and FIL study respectively. CNS prophylaxis with $12 \mathrm{mg}$ of intrathecal methotrexate (IT MTX) was administered in patients with a high risk of CNS progression/relapse according to local clinical practice. All patients received primary prophylaxis for neutropenia, with a $6 \mathrm{mg}$ pegfilgrastim subcutaneous injection on day 2 in MC078E trial and with granulocyte colony-stimulating factors in REAL07 trial. Prophylaxis for deep vein thrombosis was as follows in both trials: low dose aspirin (acetylsalicylic acid), $81 \mathrm{mg}$ per day and prophylactic low molecular weight heparins in MC078E and REAL07 trials respectively. Pneumocystis Jirovecii infection prophylaxis with co-trimoxazole or pentamidine aerosol was administered according to local clinical practice. Occult carriers of hepatitis $B$ virus were treated with lamivudine. A prephase treatment with steroids or vincristine in cases of urgent clinical need was allowed in 7 days before study treatment. Tumor lysis prophylaxis, antiemetics, and supportive care were the standard of care and at the discretion of the treating physician.

\section{Pathology review and DLBCL profile assessment}

All histologic diagnoses were confirmed by a central pathology review using WHO 2008 classification 
diagnostic criteria $^{27}$ in both trials. $\mathrm{COO}$ subtype was determined by Hans algorithm IHC as either GCB or non$\mathrm{GCB}^{15}$.

In MC078E trial, the histological diagnoses and $\mathrm{COO}$ phenotype were validated independently at the British Columbia Cancer Agency on a subset of the study cases $(N=43)$ for standardization. There was agreement with the assessment of GCB versus non-GCB in 93\% (40 of 43) of cases. As for the REAL07 trial, two pathologists centrally reviewed diagnostic lymphoma samples from each patient for IHC COO. Differences of opinion were resolved by joint review with a multihead microscope.

\section{Outcome}

Progression-free survival (PFS) was defined as the time from the date of registration until the date of disease progression or death due to any cause. Time to progression (TTP) was defined as the time from the date of registration until the date of disease progression. Overall survival (OS) was defined as the time from the date of registration until the date of death as a result of any cause. Additional analyses of the association between outcome and cell of origin and IPI were performed.

The incidence of CNS relapses, late toxicities, and secondary malignancies was reported.

Late toxicities were defined as any type of toxicity that was reported from the date of the treatment completion until the date of the last follow-up and recorded as being possibly, probably or definitely related to study treatment. Adverse events were defined as per National Cancer Institute Common Terminology Criteria for Adverse Events $\mathrm{v}$ 4.03. Toxicities were described as maximum grade occurred for each patient.

\section{Statistical methods}

The MC078E trial was a phase II study that utilized a one-stage binomial design to assess the efficacy and tolerability of R2-CHOP21 regimen with 93\% power and 9\% type I error rate. The REAL07 trial was a phase II study designed according to Simon's two stage minimax design, with $80 \%$ power and $5 \%$ type I error rate.

In the present long-term combined analysis from the two trials, all patients were analyzed as a single cohort. Long-term outcome results from the two trials separately were also reported. The distribution of time-to-event survival end points were estimated by using Kaplan Meier methods. Differences between groups were evaluated by log-rank statistics. For PFS and TTP, patients were censored on the date of their last disease assessment. For OS, patients were censored on the date of last follow-up. A subgroup analysis of PFS, TTP, and OS by COO phenotype and IPI was performed.

\section{Ethics}

The trial was done in accordance with the Declaration of Helsinki and good clinical practice guidelines. Approval was obtained from the independent ethics committees and institutional review boards at each site before trial initiation. All patients provided written informed consent to participate in the study.

\section{Results}

Between September 2008 and August 2013, 63 patients with DLBCL were enrolled in the MC078E trial and were included in the present long-term follow-up analysis. In the REAL07 phase II trial, 49 patients, enrolled between October 2008 and June 2011, were included. Hence, the entire cohort included 112 patients (63 MC078E, 49 REAL07) with de-novo DLBCL.

\section{Clinical characteristics}

The median age of the whole cohort was 69 years (range 22-87 years), with $50(44.6 \%)$ patients over age 70 years and five (4.5\%) patients over age 80. Main clinical characteristics are summarized in Table 1.

Patients characteristics included the following: 68 (60.7\%) patients; Ann Arbor advanced stage III-IV in 98 $(87.5 \%)$ cases; B symptoms in 39 (41.1\%) cases. According to IPI, patients were stratified as low/intermediate-low risk (0-2) in $49(43.8 \%)$ cases and intermediate-high/high risk (3-5) in $63(56.3 \%)$ cases. According to central nervous system (CNS)-IPI, patients were stratified as low risk CNS-IPI $0-1$ in seven (6.3\%), intermediate risk CNS-IPI $2-3$ in 82 (73.2\%) and high risk CNS IPI 4-6 in $23(20.6 \%)$ patients. Excluding $22(19.6 \%)$ patients that were not evaluable for COO IHC testing, GCB vs non-GCB was observed in $47(42.0 \%)$ vs $43(38.4 \%)$ cases respectively.

\section{Long-term follow-up outcome MCO78E trial and REALO7 trial}

At a median FU of 5.1 years (y), 5y-PFS was 59\% (95\% CI, $48-73 \%)$ vs $69 \%$ (95\% CI, 57-85\%) ( $p=0.09$ ), 5y-TTP was $68 \%$ (95\% CI, $57-81 \%$ ) vs $72 \%$ (95\% CI, $60-88 \%)(p=$ $0.24)$ and 5 y-OS was $74 \%$ (95\% CI, $63-865)$ vs $77 \%(95 \%$ $\mathrm{CI}, 64-92 \%)(p=0.28)$. Since differences in the long-term outcomes between the two trials were observed to be not statistically significant, a combined analysis of the two cohorts was done.

\section{Whole cohort}

At a median follow-up of 5.1 years (y), for the whole cohort, 5y-PFS was $63.5 \%$ (95\% CI, 54.7-73.6\%), 5y-TTP was $70.1 \%$ (95\% CI, 61.6-79.9\%) and 5y-OS was 75.4\% (95\% CI, 67.3-84.5\%) (Fig. 1). A total of 32 relapses were observed, with only 2 cases of CNS relapse. In the two patients who experienced CNS-relapse, the time from 
Table 1 Patient characteristics

MC078E $(N=63) \quad$ REAL07 $(N=49) \quad$ Total $(N=112)$

Age at diagnosis

$\begin{array}{llll}\text { N } & 63 & 49 & 112 \\ \text { Mean (SD) } & 64.5(13.24) & 69.4(5.16) & 66.7(10.74) \\ \text { Median } & 67.0 & 69.0 & 69.0 \\ \text { Range } & 22.0,87.0 & 61.0,79.0 & 22.0,87.0\end{array}$

Sex, $n(\%)$

$\begin{array}{llll}\text { Male } & 39(61.9 \%) & 29(59.2 \%) & 68(60.7 \%) \\ \text { Female } & 24(38.1 \%) & 20(40.8 \%) & 44(39.3 \%) \\ \text { Stage, } n(\%) & & & \\ \text { I/II } & 8(12.7 \%) & 6(12.2 \%) & 14(12.5 \%) \\ \text { III/IV } & 55(87.3 \%) & 43(87.8 \%) & 98(87.5 \%)\end{array}$

Systemic symptoms, $n$ (\%)

$\begin{array}{llll}\text { A } & 30(65.2 \%) & 26(53.1 \%) & 56(58.9 \%) \\ \text { B } & 16(34.8 \%) & 23(46.9 \%) & 39(41.1 \%) \\ \text { Missing } & 17 & 0 & 17 \\ \text { CNS-IPI, n (\%) } & & & \\ 1 & 6(9.5 \%) & 1(2.0 \%) & 7(6.3 \%) \\ 2 & 22(34.9 \%) & 19(38.8 \%) & 41(36.6 \%) \\ 3 & 25(39.7 \%) & 16(32.7 \%) & 41(36.6 \%) \\ 4 & 6(9.5 \%) & 10(20.4 \%) & 16(14.3 \%) \\ 5 & 4(6.3 \%) & 3(6.1 \%) & 7(6.3 \%)\end{array}$

IPI Group, $n$ (\%)

$\begin{array}{llll}0-2 & 29(46.0 \%) & 20(40.8 \%) & 49(43.8 \%) \\ 3+ & 34(54.0 \%) & 29(59.2 \%) & 63(56.3 \%) \\ \text { COO, } n(\%) & & & \\ \text { GCB } & 33(55.9 \%) & 14(45.2 \%) & 47(52.2 \%) \\ \text { Non-GCB } & 26(44.1 \%) & 17(54.8 \%) & 43(47.8 \%) \\ \text { Missing } & 4 & 18 & 22\end{array}$

Days from diagnosis to randomization

\begin{tabular}{llll}
$N$ & 63 & 49 & 112 \\
Mean (SD) & $16.9(9.38)$ & $25.5(14.72)$ & $20.7(12.71)$ \\
Median & 14.0 & 26.0 & 18.0 \\
Range & $4.0,42.0$ & $1.0,67.0$ & $1.0,67.0$ \\
\hline
\end{tabular}

IPI International Prognostic Index, CNS-IPI central nervous system-IPI, COO cell of origin, GCB germinal center B-cell

randomization to CNS-relapse was 287 and 183 days, the CNS-IPI was 4 and 3, COO was GCB and non-GCB, respectively; no intrathecal CNS phrophylaxis was administered in these two patients. Late relapse occurring beyond 3 years was observed in four cases (three cases with GCB phenotype and one case with missing COO data).

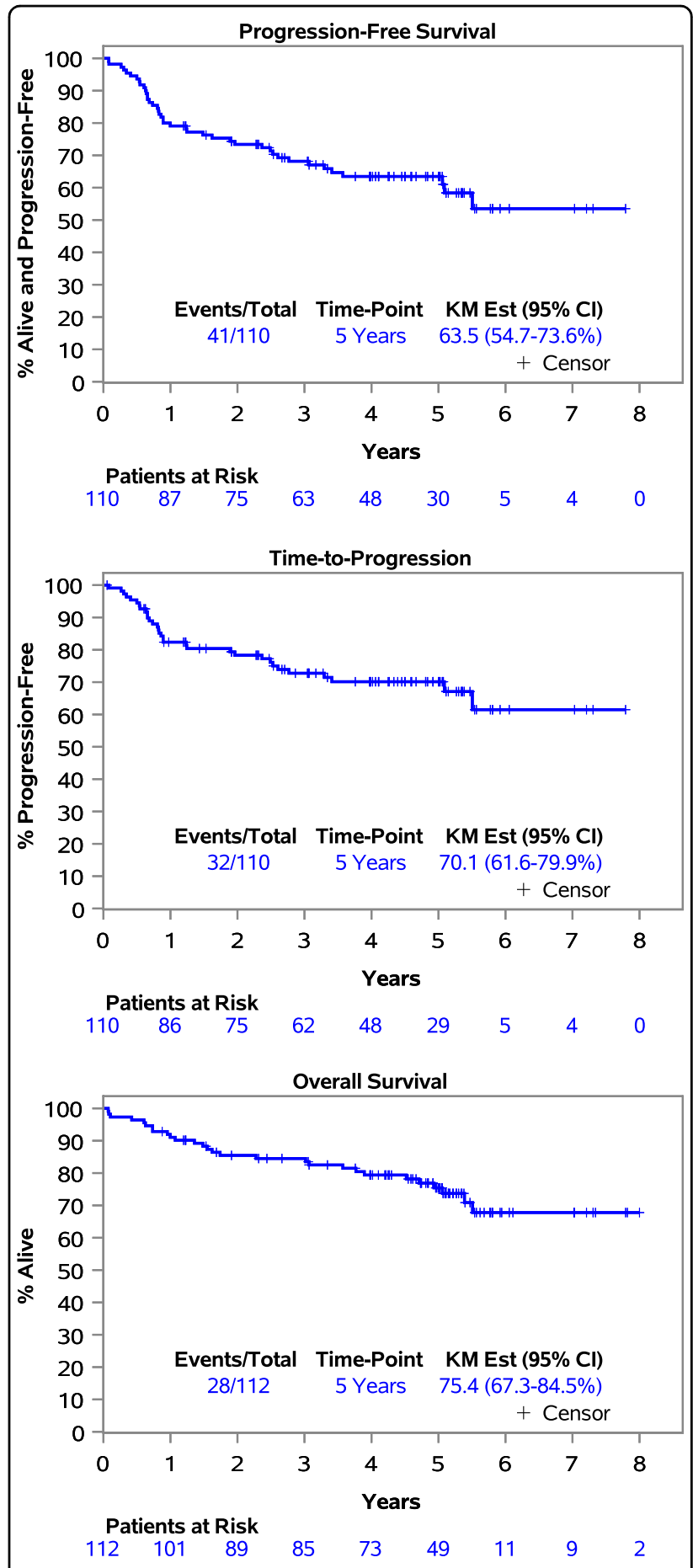

Fig. 1 Kaplan-Meier curves of progression-free survival, time to progression, overall survival of the whole cohort

Twenty-five patients died with the following causes: lymphoma in 15 (60\%) patients, late toxicities in one (4\%), second tumors in three (12\%), and other causes not related to hematological disease or treatment in six (24\%) patients (one due to violent cause, one due to diabetes 


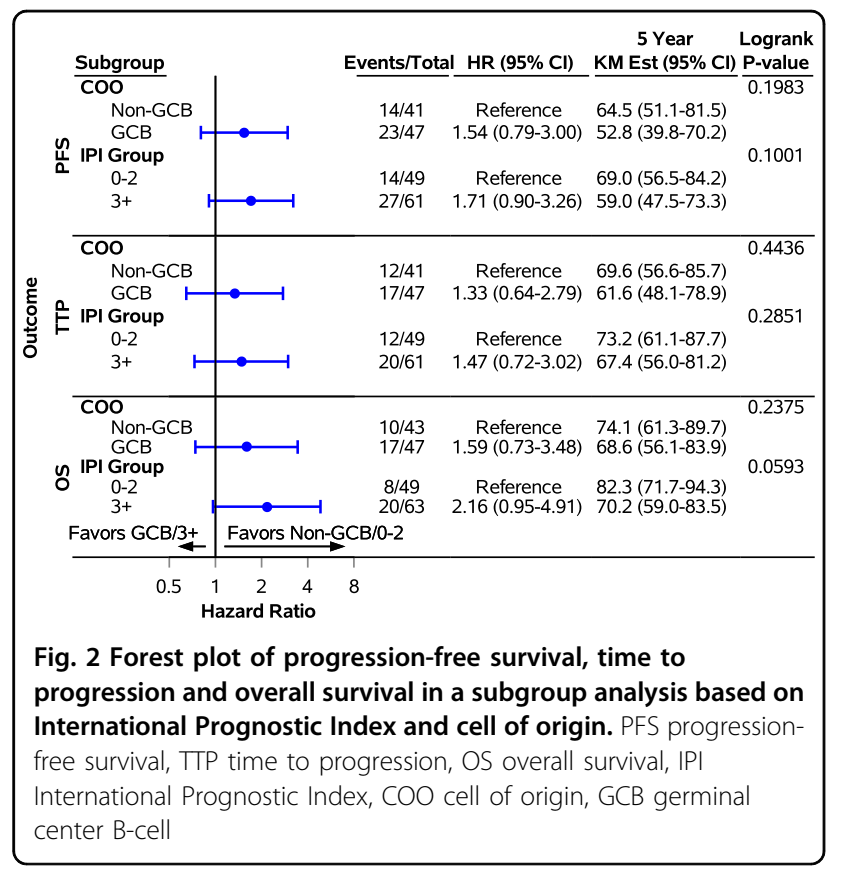

mellitus type 2 complications, three due to acute cardiorespiratory arrest and one due to bacteriemia, all nonrelated to lymphoma or treatment according to investigators).

Outcome results in a subgroup analysis stratifying patients according to COO were: $5 \mathrm{y}$-PFS was $52.8 \%$ (95\% CI, $39.8-70.2 \%)$ vs $64.5 \%$ (95\% CI, $51.1-81.5 \%) \quad(p=$ 0.198 ), 5 y-TTP was $61.6 \%$ (95\% CI, $48.1-78.9 \%$ ) vs $69.6 \%$ (95\% CI, $56.6-85.7 \%)(p=0.444)$ and $5 y-O S$ was $68.6 \%$ (95\% CI, $56.1-83.9 \%)$ vs $74.1 \%$ (95\% CI, $61.3-89.7 \%)(p=$ 0.238 ) in GCB vs non-GCB respectively (Fig. 2). Outcome results in a subgroup analysis stratifying patients according to IPI $0-2$ vs $3-5$ were: $5 y$-PFS was $69.0 \%(95 \% \mathrm{CI}$, $56.5-84.2 \%)$ vs $59.0 \%$ (95\% CI, $47.5-73.3 \%)(p=0.100)$, 5y-TTP was $73.2 \%$ (95\% CI, $61.1-87.7 \%)$ vs $67.4 \%(95 \%$ $\mathrm{CI}, 56.0-81.2 \%)(p=0.285)$ and $5 \mathrm{y}-\mathrm{OS}$ was $82.3 \%(95 \%$ CI, $71.7-94.3 \%)$ vs $70.2 \%$ (95\% CI, $59.0-83.5 \%) \quad(p=$ $0.059)$ (Figs. 2 and 3).

\section{Late toxicities and second tumors}

Only one toxic death has been recorded in the follow-up period: a grade 5 sepsis occurred 6 months after the treatment completion in patient that was not neutropenic. Three patients experienced a severe grade 4 late toxicity (all grade 4 persistent neutropenia, subsequently resolved). Other milder grade $\leq 3$ toxicities were: infections (in four cases, one grade 3 Gram negative bacteriemia and three cases of grade 1-2: viral infections in one case, Gram positive infections in two cases), thrombosis (one case grade 2) and persistent neuropathy (three cases, all grade 1-2). Two cases of cardiovascular disease grade 3 were reported.

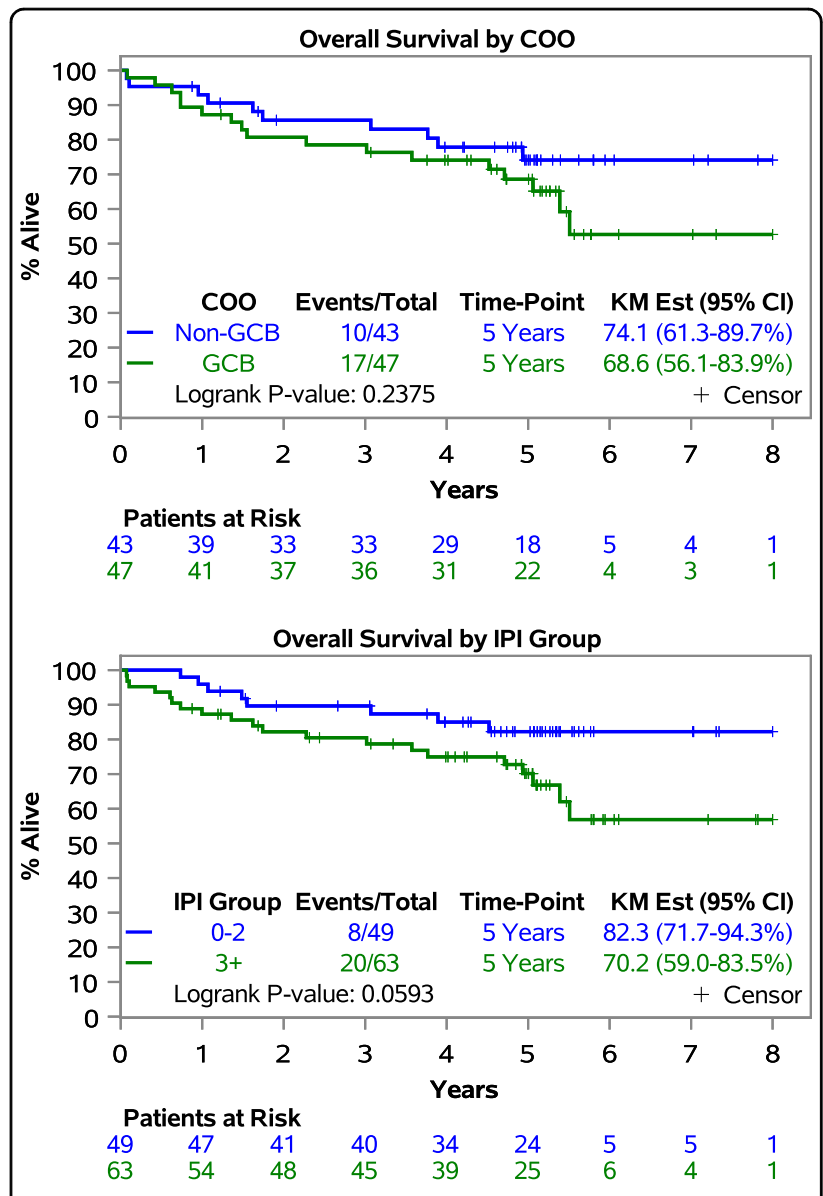

Fig. 3 Kaplan-Meier curves of overall survival in a subgroup analysis based on International Prognostic Index and cell of origin. IPI International Prognostic Index, COO cell of origin, GCB germinal center B-cell

Second malignancies were observed in seven patients (6.3\%): one (0.9\%) case of therapy-related secondary acute myeloid leukemia; six cases of other not therapy-related second tumors (two (1.8\%) cases of second lymphoma (one T-cell and one mucosa-associated lymphoid tissue (MALT) lymphoma), one metastatic adenocarcinoma of unknown origin, one prostatic cancer, one rectal adenocarcinoma, and one non-melanotic tumor of the skin). Three patients died due to the occurence of the second tumor. The median time from the end of treatment to the second neoplasia onset is 16.4 months (range: 5.7-53.3 months).

\section{Discussion}

This long-term follow-up analysis demonstrates that in patients with de novo DLBCL the R2-CHOP21 regimen maintained high efficacy over time, with high rates of PFS, TTP, and OS and with a good long-term safety profile.

At a median follow-up of 5.1 years (y), for the whole cohort, 5y-PFS was 63.5\% (95\% CI, 54.7-73.6\%), 5y-TTP 
was $70.1 \%(95 \% \mathrm{CI}, 61.6-79.9 \%)$ and $5 \mathrm{y}-\mathrm{OS}$ was $75.4 \%$ (95\% CI, 67.3-84.5\%) (Fig. 1).

These results find a favorable comparison with historical data on patients treated with standard R-CHOP. Nowakowski et al. ${ }^{22}$, in a control cohort of DLBCL patients treated with R-CHOP alone, obtained from Mayo Clinic Database, showed a 2y-PFS of 52\%; while in our present cohort treated with $\mathrm{R}-\mathrm{CHOP}$ in association to lenalidomide 5y-PFS in the whole DLBCL cohort was $63.5 \%$. These results are promising on a role of the addiction of lenalidomide to standard front-line chemoimmunotherapy in DLBCL setting.

Two randomized studies ${ }^{25,26}$ have completed accrual and are expected to be reported in the near future. E1412 is a randomized phase 2 study, which enrolled patients with newly diagnosed DLBCL regardless of COO and is powered to analyse $\mathrm{COO}$ results separately. Robust trial is a phase 3 registrational study that enrolled patients with ABC DLBCL only. These long-term efficacy and safety data in the present analysis will aid in the interpretation of the early results of these randomized trials.

In terms of efficacy, the current analysis demonstrates continuous benefit of R2CHOP with only a few relapses beyond year 2 . Interestingly, late relapses appeared to occur in the GCB subtype.

In a subgroup analysis by the clinical prognostic index IPI, we can observe that IPI remained almost significantly predictive of survival (with patients with IPI score > 3 showing inferior OS, $p=0.059$ ).

However, if we compared the outcome results of patients with intermediate-high/high IPI risk treated with R2-CHOP in our cohort, to historical data of IPI $>3$ DLBCL treated with R-CHOP standard, we observed a 3yOS of $80.5 \%$ vs $65.1 \%$ (IPI 3) and $59.0 \%$ (IPI 4-5) in patients treated with $\mathrm{R} 2-\mathrm{CHOP}$ vs historical R-CHOP treated control cohort, respectively ${ }^{28}$. These data suggested a role of association of lenalidomide to standard R$\mathrm{CHOP}$ in improving the prognosis also of intermediatehigh/high risk patients.

Both MC078E and REAL07 trials performed an exploratory analysis of patients treated with the combination regimen R2-CHOP21 in GCB vs non-GCB phenotype, suggesting that the addition of lenalidomide could mitigate the negative prognostic impact of the non-GCB subtype $^{22,24}$. In the MC078E study ${ }^{22}$, a cohort of consecutive DLBCL patients, stage II-IV, from the Mayo Clinic Lymphoma Database treated with standard RCHOP21 was retrospectively analyzed as a control. The outcome of the non-GCB phenotype was inferior to that of GCB in DLBCL patients treated with R-CHOP21 alone (2y-PFS in non-GCB vs GCB subtype of $28 \%$ vs $64 \%$, respectively, $p=0.001$ ), while there was no difference in outcome between the non-GCB vs GCB subtype in patients treated with $\mathrm{R} 2-\mathrm{CHOP} 21$ combination regimen.
The current analysis demonstrates a durable benefit of R2-CHOP in non-GCB DLBCL, while results on GCB DLBCL showed to be superimposable to what obtained with standard $\mathrm{R}-\mathrm{CHOP}^{22}$. This long-term response seems to translate into an apparent survival benefit in non-GCB DLBCL when compared to historical controls $(2 y-O S$ in R-CHOP treated cohort was $46 \%$ vs $5 \mathrm{y}-\mathrm{OS}$ of $74.1 \%$ reported in our cohort of non-GCB DLBCL patients treated with R2-CHOP). Moreover, R2-CHOP21 was effective in patients with a high-intermediate/high IPI score and an older age (44.6\% and $4.5 \%$ were over 70 and 80 years old, respectively).

A more accurate definition of $\mathrm{COO}$, if compared with IHC, could be provided by GEP analysis such as the Nanostring ${ }^{\circledR}$ platform. In order to better evaluate the role of the association of lenalidomide to standard chemoimmunotherapy in different $\mathrm{COO}$ phenotypes, Nanostring ${ }^{\circledR}$ analysis in these patients is ongoing. Other recent trials to improve front-line outcomes of DLBCL patients investigated new regimens in both GCB and non-GCB/ $\mathrm{ABC}$ subtypes. In the recent published GOYA trial ${ }^{13}$, which included more patients with a low-intermediate IPI risk score compared to the population of our study, a subgroup analysis according to COO determined by GEP was performed. A $3 y$-PFS of $75 \%, 59 \%$, and $63 \%$ for the $\mathrm{GCB}, \mathrm{ABC}$, and unclassified phenotype, respectively, was reported with no difference between the rituximab and obinotuzumab arms. When compared to the 3y-PFS of patients treated with $\mathrm{R} 2-\mathrm{CHOP}$ in our analysis, the combination of lenalidomide may influence outcomes in non-GCB subtype DLBCL (3y-PFS of $61.2 \%$ and $67.4 \%$ in GCB and non-GCB respectively). The role of the combination of lenalidomide with standard R-CHOP21 in other subgroups of DLBCL with worse prognosis, such as MYC/ BCL2 double expressors or double hit lymphomas remains to be defined, and further studies are ongoing. Future directions on the optimal use of lenalidomide toward a tailored therapy in DLBCL patients could come from the most recent advances in genetic and molecular knowledge of $\mathrm{DLBCL}^{29}$.

Despite a large number of intermediate/high CNS-IPI patients, CNS recurrences were less than expected, suggesting a role of lenalidomide combination in decreasing the risk of CNS involvement. There is evidence that small molecules, such as lenalidomide, could cross the blood-brain barrier. Lenalidomide also has been demonstrated to have single-agent activity in primary CNS lymphomas. In addition, non-GCB/ABC DLBCL are more likely to involve the CNS, and improved outcomes in this subgroup of patients could contribute to a reduced CNS-relapse rate ${ }^{30}$.

The addition of a new drug to standard chemoimmunotherapy raises concerns of increased toxicities, especially in older patients. This long-term follow-up analysis 
demonstrates that no new worrisome safety signals were observed in patients treated with an R2-CHOP21 combination regimen. In 112 patients, only one toxic death has been recorded in the follow-up period, a grade 5 sepsis that occurred 6 months after the completition of treatment. The other grade 4 late toxicities were grade 4 persistent neutropenia and all subsequently resolved. Among the four cases of infections reported, only two cases occurred in neutropenic patients (the case of grade 3 Gram negative bacteriemia and one grade 1-2 infection), and both resolved. The only patient death secondary to sepsis was not related to neutropenia at the time of the event. In keeping with earlier published results which suggested that the incidence of neuropathy was low $^{21-24}$ with R2CHOP21, long-term analysis shows only three patients reported a persistent neuropathy, all mild/grade $1-2$. Two cases of cardiovascular disease grade 3 were observed, but it must be noted that the median age of patients was high and cardiotoxicity could be attributed mainly to antracycline-based therapy.

The incidence of second malignancies was comparable rates in patients treated with $\mathrm{RCHOP}^{31}$. Moser et al., reported, for patients treated with $\mathrm{CHOP}$ or $\mathrm{CHOP}$ like therapy, a cumulative incidence at 15 years of therapyrelated secondary acute leukemia/myelodysplastic syndrome and other tumors of $3 \%$ and $11 \%$ respectively ${ }^{30}$. In our cohort, the cumulative incidence at 5 years was $0.9 \%$ and $5.4 \%$ respectively. Indeed, considering the median age of patients, prostatic cancer and tumor of the skin are quite common in this population.

In conclusion, our long-term follow-up combined analysis from two phase II trials shows that R2-CHOP21 efficacy was maintained over time with a high rate of PFS, TTP, and OS, considering high risk feature patients that were included. The addition of lenalidomide to RCHOP appears to mitigate the negative prognostic impact of non-GCB phenotype. The incidence of therapy related secondary malignancies was low, and no new worrisome long-term safety concerns were reported. Phase III randomized trials have recently concluded, with early results expected in the near future. These long-term efficacy and safety data will aid in the interpretation of the results.

\section{Acknowledgements}

Celgene provided funding for the two phase 2 studies from which the data were obtained. Celgene had no role in study design, data collection, data analysis, data interpretation, or manuscript preparation. Fondazione Italiana Linfomi.

\section{Author contributions}

A.C., A.C., T.E.W., U.V., and G.S.N.: Study design, data collection, data interpretation, manuscript writing and editing. G.C., B.R.L, L.D.P.: data analysis, data interpretation, Figures, Tables, manuscript writing and editing. G.G., W.R. M., G.I., C.B.R., A.T., R.L.K., A.C., J.M.F., V.P., C.E.R., M.S., S.M.A., F.C., A.L.M., T.M.H.: data collection, data interpretation, manuscript editing.

\begin{abstract}
Author details
${ }^{1}$ Azienda Ospedaliero Universitaria Città della Salute e della Scienza di Torino, Torino, Italy. ${ }^{2}$ Mayo Clinic, Rochester, MN, USA. ${ }^{3}$ Department of translational Medicine, University of Eastern Piedmont, Novara, Italy. ${ }^{4}$ Cornell University, New York, NY, USA. ${ }^{5}$ Mayo Clinic, Scottsdale, AZ, USA. ${ }^{6}$ Azienda Ospedaliera Spedali Civili di Brescia, Brescia, Italy. ${ }^{7}$ IRCCS Azienda Ospedaliera Universitaria San Martino - IST Istituto Nazionale per la Ricerca sul Cancro, Genova, Italy. ${ }^{8}$ Mayo Clinic, Jacksonville, FL, USA. ${ }^{9}$ Unit of Hematology and Hemopoietic Stem Cell Transplantation, Ospedale Cardinale G Panico, Tricase, Italy.

${ }^{10}$ Division of Medical Oncology, Centro di Riferimento Oncologico Aviano National Cancer Institute, Aviano, Italy. " Department of Oncology and Hematology, Infermi Hospital, Rimini, Italy. ${ }^{12}$ Unit of Clinical Epidemiology, CPO, Azienda Ospedaliero Universitaria Città della Salute e della Scienza di Torino, Torino, Italy
\end{abstract}

\section{Conflict of interest}

A.Ch.: advisory board: Celgene, Janssen; honoraria for lectures: Amgen, Celgene, Janssen, Nanostring, Roche, Teva. G.G.: consultancy: Roche, Karyopharm, Morphosys, Gilead, Janssen, Novartis; honoraria: Roche, Karyopharm, Morphosys, Gilead, Janssen, Novartis; speakers bureau: Roche, Gilead, Janssen, Novartis. J.M.F.: Celgene grants during the study conductions. F.C.: honoraria: Celgene, Onyx, Janssen. T.E.W.: honoraria from Celgene, personal fees from Seattle genetics, outside the submitted work. U.V.: advisory board: Roche, Celgene, Janssen; honoraria for lectures: Roche, Celgene, Takeda, Gilead, Janssen, Mundipharma; research funding: Roche, Celgene. G.N.: consultancy: Bayer; research funding: Bayer, Celgene, Morphosys, Nanostring tecnologies. The remaining authors declare that they have no conflict of interest

\section{Publisher's note}

Springer Nature remains neutral with regard to jurisdictional claims in published maps and institutional affiliations.

Received: 28 June 2018 Revised: 12 September 2018 Accepted: 25 September 2018

Published online: 08 November 2018

\section{References}

1. Coiffier, B. et al. CHOP chemotherapy plus rituximab compared with CHOPalone in elderly patients with diffuse large-B-cell lymphoma. New Engl. J. Med. 346, 235-242 (2002).

2. Habermann, T. M. et al. Rituximab-CHOP versus CHOP alone or with maintenance rituximab in older patients with diffuse large B-cell lymphoma. J. Clin. Oncol. 24, 3121-3127 (2006).

3. Pfreundschuh, M. et al. CHOP-like chemotherapy plus rituximab versus CHOPlike chemotherapy in young patients with good-prognosis diffuse large B-cell lymphoma: a randomized controlled trial by the MabThera International Trial (MlnT) Group. Lancet Oncol. 7, 379-391 (2006).

4. Sehn, L. H. et al. Introduction of combined CHOP plus rituximab therapy dramatically improved outcome of diffuse large B-cell lymphoma in British Columbia. J. Clin. Oncol. 23, 5027-5033 (2005).

5. Farooq, U. et al. Clinical heterogeneity of diffuse large B-cell lymphoma following failure of front-line immunochemotherapy. Br. J. Haematol. 179, 50-60 (2017).

6. Maurer, M. J. et al. Event-free survival at 24 months is a robust end point for disease-related outcome in diffuse large B-cell lymphoma treated with immunochemotherapy. J. Clin. Oncol. 32, 1066-1073 (2014).

7. Delarue, R. et al. R-CHOP14 compared to R-CHOP21 in elderly patients with diffuse large B cell lymphoma: results of the interim analysis of the LNHO3-6B GELA study. Blood 114, 169 (2009). [abstract 406].

8. Chiappella, A. et al. Rituximab-dose-dense chemotherapy with or without high-dose chemotherapy plus autologous stem-cell transplantation in highrisk diffuse large B-cell lymphoma (DLCL04): final results of a multicentre, open-label, randomised, controlled, phase 3 study. Lancet Oncol. 18, 1078-1088 (2017).

9. Schmitz, N. et al. German High-Grade Lymphoma Study Group (DSHNHL). Conventional chemotherapy (CHOEP-14) with rituximab or high-dose 
chemotherapy (MegaCHOEP) with rituximab for young, high-risk patients with aggressive B-cell lymphoma: an open-label, randomised, phase 3 trial (DSHNHL 2002-1). Lancet Oncol. 13, 1250-1259 (2012).

10. Stiff, P. J. et al. Autologous transplantation as consolidation for aggressive nonHodgkin's lymphoma. N. Engl. J. Med. 369, 1681-1690 (2013).

11. Dholaria, B. et al. DA.R-EPOCH vs. R-CHOP for high-risk diffuse large B- cell lymphoma: The Mayo Clinic Florida Experience. Blood 130, 1572 (2017).

12. Lugtenburg, P. J. et al. Randomized phase III study on the effect of early intensification of rituximab in combination with 2-weekly CHOP chemotherapy followed by rituximab or no maintenance in patients with diffuse large Bcell lymphoma: Results from a HOVON-Nordic Lymphoma Group study. JCO.2016.34.15_suppl.7504.

13. Vitolo, U. et al. Obinutuzumab or rituximab plus cyclophosphamide, doxorubicin, vincristine, and prednisone in previously untreated diffuse large B-cell lymphoma. J. Clin. Oncol. 35, 3529-3537 (2017).

14. Alizadeh, A. A. et al. Prediction of survival in diffuse large B-cell lymphoma based on the expression of 2 genes reflecting tumor and microenvironment. Blood 118, 1350-1358 (2011).

15. Hans, C. P. et al. Confirmation of the molecular classification of diffuse large Bcell lymphoma by immunohistochemistry using a tissue microarray. Blood 103, 275-282 (2004)

16. Choi, W. W. et al. A new immunostain algorithm classifies diffuse large B-cell lymphoma into molecular subtypes with high accuracy. Clin. Cancer Res. 15 , 5494-5502 (2009).

17. Rosenwald, A. et al. The use of molecular profiling to predict survival after chemotherapy for diffuse large B-cell lymphoma. N. Engl. J. Med. 346 1937-1947 (2002)

18. Czuczman, M. S. et al. A phase $2 / 3$ multicenter, randomized study comparing the efficacy and safety of lenalidomide versus investigator's choice in relapsed/refractory DLBCL. Clin. Cancer Res. 16, 2818 (2017).

19. Hernandez-llizaliturri, F. J. et al. Higher response to lenalidomide in relapsed/ refractory diff use large B-cell lymphoma in nongerminal center B-cell-like than in germinal center B-cell-like phenotype. Cancer 117, 5058-5066 (2011).

20. Zhang, L. H. et al. Lenalidomide efficacy in activated b-cell-like subtype diffuse large B-cell lymphoma is dependent upon IRF4 and cereblon expression. Br. J. Haematol. 160, 487-502 (2013).
21. Nowakowski, G. S. et al. Lenalidomide can be safely combined with R-CHOP (R2CHOP) in the initial chemotherapy for aggressive B-cell lymphomas: phase I study. Leukemia 25, 1877-1881 (2011).

22. Nowakowski, G. S. et al. Lenalidomide combined with R-CHOP overcomes negative prognostic impact of non-germinal center B-cell phenotype in newly diagnosed diffuse large B-Cell lymphoma: A Phase II Study. J. Clin. Oncol. 33, 251-257 (2014).

23. Chiappella, A. et al. Lenalidomide plus cyclophosphamide, doxorubicin, vincristine, prednisone and rituximab is safe and eff ective in untreated, elderly patients with diff use large B-cell lymphoma: phase I study by the Fondazione Italiana Linfomi. Haematologica 98, 1732-1738 (2013).

24. Vitolo, U. et al. Lenalidomide plus R-CHOP21 in elderly patients with untreated diff use large B-cell lymphoma: results of the REAL07 open-label, multicentre phase 2 trial. Lancet Oncol. 15, 730-737 (2014).

25. Nowakowski, G. S. et al. ROBUST: lenalidomide-R-CHOP versus placebo-R$\mathrm{CHOP}$ in previously untreated ABC-type diffuse large B-cell lymphoma. Future Oncol. 12, 1553-1563 (2016). (ClinicalTrial.gov Identifier NCT02285062).

26. King, R. L. et al. Rapid, real time pathology review for ECOG/ACRIN 1412: a novel and successful paradigm for future lymphoma clinical trials in the precision medicine era. Blood Cancer J. 8, 27 (2018) (ClinicalTrial.gov Identifier NCT01856192).

27. Campo, E. et al. The 2008 WHO classification of lymphoid neoplasms and beyond evolving concepts and practical applications. Prepublished Online 117, 5019-5032 (2011).

28. Ziepert, M. et al. Standard International prognostic index remains a valid predictor of outcome for patients with aggressive CD20+ B-cell lymphoma in the rituximab era. J. Clin. Oncol. 29, 779 (2011).

29. Schmitz, R. et al. Genetics and pathogenesis of diffuse large B-cell lymphoma. NEM 378, 1396-1407 (2018)

30. Ayed, A. O. et al. CNS relapse in patients with DLBCL treated with lenalidomide plus R-CHOP (R2CHOP): analysis from two phase 2 studies. Blood Cancer J. 8, 63 (2018).

31. Moser, E. C. et al. Risk of second cancer after treatment of aggressive nonHodgkin's lymphoma; an EORTC cohort study. Haematologica 91, 1481-1488 (2006). 